\title{
Dilution of Precision Relationship between Time Difference of Arrival and Time of Arrival Techniques with No Receiver Clock Bias
}

\author{
Heon Ho Choi*, Mi Hyun Jin**, Deok Won Lim*, Sang Jeong Lee** and Chansik Park ${ }^{\dagger}$ \\ Abstract - Dilution of Precision (DOP), as a measure of positioning accuracy, is an essential factor. \\ Therefore, the DOP relationship between systems is very important. In this paper, the DOP \\ relationship between TDOA and TOA in systems lacking clock bias is derived analytically and \\ verified experimentally. Also, using those of earlier studies, the DOP relationship in each of defined \\ systems is derived analytically.
}

Keywords: DOP, TDOA, TOA, A measure of positioning accuracy

\section{Introduction}

Time of arrival (TOA) and time difference of arrival (TDOA) techniques are applied in most positioning methods, including GPS and wireless network-based locator systems [1], with the choice between them depending on factors such as transmitter structure. In TDOA, the receiver measures differences in transmission time between the master and slave stations, whereas only the respective station transmission times are measured in TOA. Furthermore, clock bias, which is included in all TOA measurements, is eliminated between transmitter and receiver in TDOA at the cost of eliminating one measurement, i.e., TDOA reduces the number of measurements for a given system by one relative to TOA.

Because the radio navigation position error is affected by several factors, it is not easy to analyze the performance of positioning algorithms. As a measure of positioning accuracy, the dilution of precision (DOP) [2], a widely used and well-studied performance measure based on the geometric arrangement of transmitters and receivers, can be multiplied by the user equivalent range error (UERE). There are three general types of measurement system for which TOA and / or TDOA can be used to analyze performance: ultrasound positioning (USP); long range navigation (LORAN); and methods using the global navigation satellite system (GNSS), in which receiver clock bias is included. Shin [1] theoretically derived a DOP relationship between TOA and TDOA in GNSS-based navigation for which they proved that, if receiver clock bias is included in the measurements, the positional DOP (PDOP) of TOA is equivalent to the DOP of TDOA. Nielsen [3,4] and Teunissen [5] determined the DOP

$\dagger$ Corresponding Author: Dept. of Electronic Engineering, Chungbuk National University, Korea. (chansp@cbnu.ac.kr)

* Dept. of Satellite Navigation System, Korea Aerospace Research Institute, Korea. (heonho@kari.re.kr)

** Dept. of Electronic Engineering, Chungnam National University, Korea. (haryane@cnu.ac.kr)

Received: January 14, 2015; Accepted: December 10, 2015 relationship between TDOA and TOA in LORAN systems, in which each measurement is independent of all others; they proved that the performance of TDOA exceeds that of TOA when four or more measurements are taken. In USP measurement, which is used for indoor navigation systems such as Cricket [6], Active Bat [7], and smart remote controllers [8], receiver clock bias is eliminated because, in addition to an ultra sound ranging signal, infrared (IR) or radio frequency (RF) signals are used to synchronize the transmitter and receiver. However, the DOP relationship between TOA and TDOA in receiver clock bias-free systems such as these has not yet been clearly determined. In this paper, the DOP relationship between TDOA and TOA in systems lacking clock bias is derived analytically and verified experimentally. These results, as well as those of earlier studies, are used to further analyze the general DOP relationship between TOA and TDOA techniques.

\section{DOP Relationship between TDOA and TOA with no Receiver Clock Bias}

\subsection{DOP in TOA}

By linearizing receiver lock bias-free measurements taken at a nominal position, a linearized measurement equation can be derived [2]:

$$
\left[\begin{array}{c}
\delta \Psi_{1} \\
\vdots \\
\delta \Psi_{n}
\end{array}\right]=\left[\begin{array}{c}
h_{1} \\
\vdots \\
h_{n}
\end{array}\right] \delta x+\left[\begin{array}{c}
v_{1} \\
\vdots \\
v_{n}
\end{array}\right] \in \mathbf{R}^{(n \times 1)},
$$

This can be rendered in vector-matrix form as

$$
\delta \boldsymbol{\Psi}=\mathbf{H} \delta x+\mathbf{v}
$$

where $\delta \boldsymbol{\Psi}$ is the error vector between the pseudorange measurements and computed ranges, $\mathbf{H}$ is the measure- 
ment matrix, and $\mathbf{v}$ is measured additive white Gaussian noise (AWGN) in the form

$$
\mathbf{v} \sim N\left(0, \sigma^{2} I\right) .
$$

Using a weighted least squares (WLSQ) algorithm, position can be estimated as [8]

$$
\delta \hat{x}=\left(\mathbf{H}^{T} \mathbf{H}\right)^{-1} \mathbf{H}^{T} \delta \boldsymbol{\Psi}
$$

with a covariance of

$$
\operatorname{cov}(\delta \hat{x})=\sigma^{2}\left(\mathbf{H}^{T} \mathbf{H}\right)^{-1}
$$

In TOA systems, the DOP is defined as [2]

$$
D O P_{T O A}=\sqrt{\operatorname{trace}\left(\mathbf{H}^{T} \mathbf{H}\right)^{-1}}
$$

where trace represents the trace of a matrix.

\subsection{DOP in TDOA}

Because TDOA measurements can be obtained by differentiating TOA measurements, the TDOA measurement equation can be represented as

$$
S_{D}\left[\begin{array}{c}
\delta \Psi_{1} \\
\vdots \\
\delta \Psi_{n}
\end{array}\right]=S_{D}\left[\begin{array}{c}
h_{1} \\
\vdots \\
h_{n}
\end{array}\right] \delta x+S_{D}\left[\begin{array}{c}
v_{1} \\
\vdots \\
v_{n}
\end{array}\right] \in \mathbf{R}^{(n-1) \times 1},
$$

in which the single difference operator $S_{D}$ is defined as

$$
S_{D}=\left[\begin{array}{cccccc}
1 & -1 & 0 & & 0 & 0 \\
1 & 0 & -1 & & 0 & 0 \\
& & & \ddots & & \\
1 & 0 & 0 & & 0 & -1
\end{array}\right] \in \mathbf{R}^{(n-1) \times n}
$$

Eq. (7) can be alternatively represented as

$$
\left[\begin{array}{c}
\delta \Psi_{1}-\delta \Psi_{2} \\
\vdots \\
\delta \Psi_{1}-\delta \Psi_{n}
\end{array}\right]=\left[\begin{array}{c}
h_{1}-h_{2} \\
\vdots \\
h_{1}-h_{n}
\end{array}\right] \delta x+\left[\begin{array}{c}
v_{1}-v_{2} \\
\vdots \\
v_{1}-v_{n}
\end{array}\right] \in \mathbf{R}^{(n-1) \times 1}
$$

Using $\mathrm{S}_{\mathrm{D}}$, (9) can in turn be represented as

$$
\delta \boldsymbol{\Psi}_{S D}=\mathbf{H}_{S D} \delta x+\mathbf{v}_{S D}
$$

where $\mathbf{v}_{S D}$ is a measurement noise term given by

$$
\mathbf{v}_{S D}=S_{D} \mathbf{v} \sim N\left(0, \sigma^{2} S_{D} S_{D}^{T}\right)=N\left(0, \sigma^{2} Q_{D}\right)
$$

The position can be estimated by using WLSQ as

$$
\delta \hat{x}=\left(\mathbf{H}_{S D}^{T} Q_{D}^{-1} \mathbf{H}_{S D}\right)^{-1} \mathbf{H}_{S D}^{T} Q_{D}^{-1} \delta \boldsymbol{\Psi}_{S D}
$$

with covariance

$$
\operatorname{cov}(\delta \hat{x})=\sigma^{2}\left(\mathbf{H}_{S D}^{T} Q_{D}^{-1} \mathbf{H}_{S D}\right)^{-1}
$$

Using (10) and (11), Eq. (13) can be represented as

$$
\begin{aligned}
\operatorname{cov}(\delta \hat{x}) & =\sigma^{2}\left(\mathbf{H}^{T} S_{D}^{T}\left(S_{D} S_{D}^{T}\right)^{-1} S_{D} \mathbf{H}\right)^{-1} \\
& =\sigma^{2}\left(\mathbf{H}^{T} S_{D}^{T} Q_{D}^{-1} S_{D} \mathbf{H}\right)^{-1}
\end{aligned}
$$

Finally, the TDOA DOP can be obtained as

$$
D O P_{T D O A}=\sqrt{\operatorname{trace}\left(\mathbf{H}^{T} S_{D}^{T} Q_{D}^{-1} S_{D} \mathbf{H}\right)^{-1}} .
$$

\subsection{DOP relationship between the TOA and TDOA techniques}

By comparing Eq. (6) with Eq. (15), the DOP relationship between TOA and TDOA techniques can be analyzed. Because

$$
Q_{D}=S_{D} S_{D}^{T}=\left[\begin{array}{llllll}
2 & 1 & 1 & & 1 & 1 \\
1 & 2 & 1 & & 1 & 1 \\
& & & \ddots & & \\
1 & 1 & 1 & & 1 & 2
\end{array}\right]=I+r r^{T} \in \mathbf{R}^{(n-1) \times(n-1)}
$$

where $r=\left[\begin{array}{lll}1 & \cdots & 1\end{array}\right]^{T} \in \mathbf{R}^{(n-1) \times 1}, Q_{D}^{-1}$ can be represented as

$$
\begin{gathered}
Q_{D}^{-1}=\left(I+r r^{T}\right)^{-1}=I-\frac{r r^{T}}{n} \in \mathbf{R}^{(n-1) \times(n-1)} \\
S_{D}^{T} Q_{D}^{-1} S_{D}=S_{D}^{T}\left(I-\frac{r r^{T}}{n}\right) S_{D}=\left(I-\frac{r_{1} r_{1}^{T}}{n}\right) \in \mathbf{R}^{n \times n}
\end{gathered}
$$

where $r_{1}=\left[\begin{array}{lll}1 & \cdots & 1\end{array}\right]^{T} \in \mathbf{R}^{n \times 1}$.

To compare $D O P_{T O A}$ and $D O P_{T D O A}$, the matrix $M_{T O A}^{U S P}$ is defined in (19) and the matrix $M_{T D O A}^{U S P}$ is defined in (20) and modified using Eq. (18):

$$
\begin{aligned}
M_{T O A}^{U S P}=\left(\mathbf{H}^{T} \mathbf{H}\right)^{-1} \\
M_{T D O A}^{U S P}=\left(\mathbf{H}^{T} S_{D}^{T}\left(S_{D} S_{D}^{T}\right)^{-1} S_{D} \mathbf{H}\right)^{-1} \\
=\left(\mathbf{H}^{T} S_{D}^{T}\left(I-\frac{r r^{T}}{n}\right) S_{D} \mathbf{H}\right)^{-1} \\
=\left(\mathbf{H}^{T} S_{D}^{T} S_{D} \mathbf{H}\right)^{-1} \\
+\left\{\frac{\left(\mathbf{H}^{T} S_{D}^{T} S_{D} \mathbf{H}\right)^{-1} \mathbf{H}^{T} S_{D}^{T} r_{1} r_{1}^{T} S_{D} \mathbf{H}\left(\mathbf{H}^{T} S_{D}^{T} S_{D} \mathbf{H}\right)^{-1}}{n-r_{1}^{T} S_{D} \mathbf{H}\left(\mathbf{H}^{T} S_{D}^{T} S_{D} \mathbf{H}\right)^{-1} \mathbf{H}^{T} S_{D}^{T} r_{1}}\right\}
\end{aligned}
$$

As $M_{T D O A}^{U S P}$ is identical to $M_{T O A}^{G N S S}$ when clock bias is 
present [1], $M_{T D O A}^{U S P}$ can be modified as

$$
M_{T D O A}^{U S P}=\left(\mathbf{H}^{T} \mathbf{H}\right)^{-1}+\frac{\left(\mathbf{H}^{T} \mathbf{H}\right)^{-1} \mathbf{H}^{T} r r^{T} \mathbf{H}\left(\mathbf{H}^{T} \mathbf{H}\right)^{-1}}{n-r^{T} \mathbf{H}\left(\mathbf{H}^{T} \mathbf{H}\right)^{-1} \mathbf{H}^{T} r} .
$$

Based on (19) and (21), Eq. (22) is satisfied at all times:

$$
\begin{aligned}
M_{T D O A}^{U S P} & =\left(\mathbf{H}^{T} \mathbf{H}\right)^{-1}+\frac{\left(\mathbf{H}^{T} \mathbf{H}\right)^{-1} \mathbf{H}^{T} r r^{T} \mathbf{H}\left(\mathbf{H}^{T} \mathbf{H}\right)^{-1}}{n-r^{T} \mathbf{H}\left(\mathbf{H}^{T} \mathbf{H}\right)^{-1} \mathbf{H}^{T} r} \\
& =M_{T O A}^{U S P}+\frac{r^{T} \mathbf{H}\left(\mathbf{H}^{T} \mathbf{H}\right)^{-2} \mathbf{H}^{T} r}{n-r^{T} \mathbf{H}\left(\mathbf{H}^{T} \mathbf{H}\right)^{-1} \mathbf{H}^{T} r} \geq M_{T O A}^{U S P}
\end{aligned}
$$

In [9], the denominator of the second term is a parameter which represents TDOP and DOP is basically positive. Also the first term and the numerator of the second term are squares form. Therefore, as shown in (22), the DOP of TDOA is always larger than that of a TOA system without clock bias by $\frac{r^{T} \mathbf{H}\left(\mathbf{H}^{T} \mathbf{H}\right)^{-2} \mathbf{H}^{T} r}{n-r^{T} \mathbf{H}\left(\mathbf{H}^{T} \mathbf{H}\right)^{-1} \mathbf{H}^{T} r}$, representing the penalty incurred by TDOA in cases where the difference operation is used to eliminate nonexistent receiver clock bias. The term $\frac{r^{T} \mathbf{H}\left(\mathbf{H}^{T} \mathbf{H}\right)^{-2} \mathbf{H}^{T} r}{n-r^{T} \mathbf{H}\left(\mathbf{H}^{T} \mathbf{H}\right)^{-1} \mathbf{H}^{T} r}$ can also be interpreted as the performance degradation resulting from the fact that one less measurement is taken in TDOA. In [1] it was shown that, if a receiver clock is used, the PDOP of TOA will be identical to the DOP of TDOA; however, this implies that TOA will be preferred in applications where receiver clock bias is absent, as it can provide more precise positioning than TDOA.

\subsection{Simulation result}

In order to verify the results derived above, a simulation was performed using MATLAB. DOPs were computed for a set of transmitters andx receivers placed randomly within a three-dimensional space as the number of receivers was increased from four to 20. It is seen from Fig. 1 , in which the differences in DOP produced by the respective techniques are plotted, that the DOP of TDOA was larger than that of TOA in all cases.

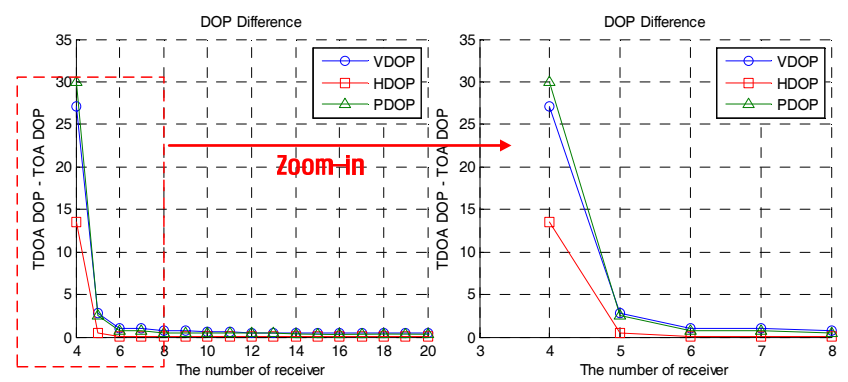

Fig. 1. DOP differences between TOA and TDOA techniques

\section{Generalized DOP Relationship between TOA and TDOA}

Based on earlier studies and the results described above, the DOP relationships for TOA and TDOA can be classified for three major measurement types, i.e., GNSS, USP, and LORAN-type measurement.

\subsection{GNSS-type measurement}

In GNSS-type measurement, clock bias is included in the measurement in order to produce a linearized measurement equation in vector-matrix form given by

$$
\begin{array}{cl}
\text { TOA }: & \delta \boldsymbol{\Psi}=\left[\begin{array}{ll}
\mathbf{H} & r
\end{array}\right]\left[\begin{array}{l}
x \\
B
\end{array}\right]+\mathbf{v} \\
T D O A: & S_{D} \delta \boldsymbol{\Psi}=S_{D} \mathbf{H} x+S_{D} \mathbf{v} .
\end{array}
$$

From (23), the DOPs of TOA and TDOA can be represented using (24) and (25), respectively [1]:

$$
\begin{aligned}
M_{T O A}^{G N S S}=\left[\mathbf{H}^{T}\left(1-\frac{r r^{T}}{n}\right) \mathbf{H}\right]^{-1} & \left(\mathbf{H}^{T} \mathbf{H}\right)^{-1}+\frac{\left(\mathbf{H}^{T} \mathbf{H}\right)^{-1} \mathbf{H}^{T} r r^{T} \mathbf{H}\left(\mathbf{H}^{T} \mathbf{H}\right)^{-1}}{n-r^{T} \mathbf{H}\left(\mathbf{H}^{T} \mathbf{H}\right)^{-1} \mathbf{H}^{T} r} \\
M_{T D O A}^{G N S S}= & {\left[\mathbf{H}^{T} S_{D}{ }^{T}\left(S_{D} S_{D}{ }^{T}\right)^{-1} S_{D} \mathbf{H}\right]^{-1} } \\
= & \left(\mathbf{H}^{T} S_{D}{ }^{T} S_{D} \mathbf{H}\right)^{-1} \\
& +\frac{\left(\mathbf{H}^{T} S_{D}{ }^{T} S_{D} \mathbf{H}\right)^{-1} \mathbf{H}^{T} S_{D}{ }^{T} r_{1} r_{1}^{T} S_{D} \mathbf{H}\left(\mathbf{H}^{T} S_{D}{ }^{T} S_{D} \mathbf{H}\right)^{-1}}{n-r_{1}^{T} S_{D} \mathbf{H}\left(\mathbf{H}^{T} S_{D}{ }^{T} S_{D} \mathbf{H}\right)^{-1} \mathbf{H}^{T} S_{D}{ }^{T} r_{1}}
\end{aligned}
$$

In [1], equations (24) and (25) were identical for GNSStype navigation measurements that included clock bias.

\subsection{USP-type measurement}

USP, which is used in network-based systems, utilizes clock bias-free measurement. Based on the results derived previously in this paper, the USP linearized measurement equation can be given in vector-matrix form as

$$
\begin{gathered}
\text { TOA }: \quad \delta \boldsymbol{\Psi}=\mathbf{H} x+\mathbf{v} \\
\text { TDOA: } \quad S_{D} \delta \boldsymbol{\Psi}=S_{D} \mathbf{H} x+S_{D} \mathbf{v} .
\end{gathered}
$$

Using (26), equations (24) and (25) can then be rewritten as

$$
\begin{gathered}
M_{T O A}^{U S P}=\left(\mathbf{H}^{T} \mathbf{H}\right)^{-1} \\
M_{T D O A}^{U S P}=\left[\mathbf{H}^{T} S_{D}{ }^{T}\left(S_{D} S_{D}{ }^{T}\right)^{-1} S_{D} \mathbf{H}\right]^{-1}
\end{gathered}
$$

As (28) is equivalent to (25) whether or not there is receiver clock bias, (28) is also equivalent to (24). Based on (24)-(28), therefore, the performance of the TOA 
technique is superior to that of the TDOA technique in receiver clock bias-free systems. This performance differential can be measured by

$$
M_{T D O A}^{U S P}-M_{T O A}^{U S P}=\frac{\left(\mathbf{H}^{T} \mathbf{H}\right)^{-1} \mathbf{H}^{T} r r^{T} \mathbf{H}\left(\mathbf{H}^{T} \mathbf{H}\right)^{-1}}{m-r^{T} \mathbf{H}\left(\mathbf{H}^{T} \mathbf{H}\right)^{-1} \mathbf{H}^{T} r}
$$

\subsection{Loran-type measurement}

In LORAN systems, each measurement has an independent form as defined in [3] and [4]. The linearized measurement equation in vector-matrix form for LORAN is given by

$$
\text { TDOA : } \quad \delta \boldsymbol{\Psi}=S_{D} \mathbf{H} x+\mathbf{v}
$$

From (30), the equation for DOP in TDOA can be represented as [3, 4]

$$
M_{T D O A}^{\text {Loran }}=\left[\mathbf{H}^{T} S_{D}^{T} S_{D} \mathbf{H}\right]^{-1}
$$

In [3] and [4], the value of Eq. (31) was less than or equal to the DOP in TOA (Eq. (23)) of a GNSS-type system with clock bias. From (27) - (31), the difference between the DOP in TOA (Eq. (28)) of a clock bias-free system and (31) is given as

$$
\begin{gathered}
\frac{\left(\mathbf{H}^{T} S_{D}{ }^{T} S_{D} \mathbf{H}\right)^{-1} \mathbf{H}^{T} S_{D}{ }^{T} r_{1} r_{1}^{T} S_{D} \mathbf{H}\left(\mathbf{H}^{T} S_{D}{ }^{T} S_{D} \mathbf{H}\right)^{-1}}{m-r_{1}^{T} S_{D} \mathbf{H}\left(\mathbf{H}^{T} S_{D}{ }^{T} S_{D} \mathbf{H}\right)^{-1} \mathbf{H}^{T} S_{D}{ }^{T} r_{1}} \\
-\frac{\left(\mathbf{H}^{T} \mathbf{H}\right)^{-1} \mathbf{H}^{T} r r^{T} \mathbf{H}\left(\mathbf{H}^{T} \mathbf{H}\right)^{-1}}{m-r^{T} \mathbf{H}\left(\mathbf{H}^{T} \mathbf{H}\right)^{-1} \mathbf{H}^{T} r} .
\end{gathered}
$$

\section{Conclusions}

Based partly on the results of earlier work, this study analyzed the DOP relationship between the TOA and TDOA techniques. An analytic DOP relationship between receiver clock bias-free TOA and TDOA techniques was derived and then evaluated through computer simulation. It was determined that the DOP of TDOA is always larger than that of TOA by $\frac{r^{T} \mathbf{H}\left(\mathbf{H}^{T} \mathbf{H}\right)^{-2} \mathbf{H}^{T} r}{n-r^{T} \mathbf{H}\left(\mathbf{H}^{T} \mathbf{H}\right)^{-1} \mathbf{H}^{T} r}$. From this, it was concluded that the performance of TOA techniques are better than those of TDOA techniques for systems without receiver clock bias. Additionally, the term $\frac{r^{T} \mathbf{H}\left(\mathbf{H}^{T} \mathbf{H}\right)^{-2} \mathbf{H}^{T} r}{n-r^{T} \mathbf{H}\left(\mathbf{H}^{T} \mathbf{H}\right)^{-1} \mathbf{H}^{T} r}$ could be interpreted as both the penalty paid by TDOA for performing a difference operation to eliminate receiver clock bias in the absence of such bias and as the result of reducing the number of measurements by one. Finally, using results of this study with those of earlier studies, the DOP relationship in each of defined systems is derived analytically.

\section{References}

[1] Dongho Shin, Taekyung Sung, "Comparisons of error characteristics between TOA and TDOA positioning," IEEE Trans. Aerospace and Electronic Systems, vol. 38, no. 1, pp. 307-311, Jan. 2002.

[2] Elliott D. Kaplan and Christopher Hegarty, Understanding GPS Principles and Applications: Artech House, Norwood, MA, 2006.

[3] Nilsen, R. O., "Relationship between dilution of precision for point positioning and for relative positioning with GPS," IEEE Trans. Aerospace and Electronic Systems, vol. 33, no. 1, pp. 333-337, Jan. 1997.

[4] Park, C., and Kim, I., Comments on "Relationship between dilution of precision for point positioning and for relative positioning with GPS," IEEE Trans. Aerospace and Electronic Systems, vol. 36, no. 1, pp. 73-74, Jan. 2000.

[5] Teunissen, P. J. G., “A proof of Nielsen's conjecture on the GPS dilution of precision," IEEE Trans. Aerospace and Electronic Systems, vol. 34, no. 2, pp. 693-695, Apr. 1998.

[6] Priyantha, N., Chakraborty, A. and Balakrishnan, H., "The Cricket Location-Support System," in Proceedings of 6th ACM MOBICOM Conference, Boston, MA, USA, Aug. 2000.

[7] Oliver, J., and Robert K. H., "Concurrent scheduling in the Active Bat location system," in Proceedings of the 6th IEEE International Workshop on Sensor Networks and Systems for Pervasive Computing, Mannheim, Germany, Mar. 2010.

[8] E. W. Kamen, and J. K. Su, Introduction to Optimal Estimation: Springer, London, MA, 1996.

[9] Sung-Hoon Yang, Chang Bok Lee, Moon Beom Heo, Eunsung Lee, Sang Jeong Lee, and Chansik Park, "Analysis of a clock-aided global navigation satellite system(GNSS)," Meas. Sci. Technol., vol. 22, no. 3, pp. 35104-35110, Mar. 2011.

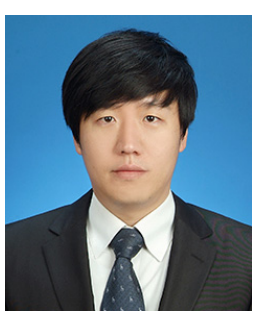

Heon Ho Choi He is a senior research engineer of the Satellite Navigation Team at the Korea Aerospace Research Institute, Korea. He received B.S., M.S and Ph.D. degrees in Electronics Engineering Department from Chungnam National University. His research interests are the direction finding algorithm and signal processing. 


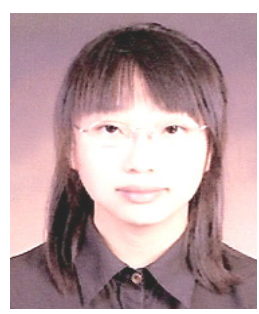

Mi Hyun Jin She is a Ph.D. student at the Electronics Engineering Department of Chungnam National University, Korea. Her current research interests are the signal abnormality detection and anti-jamming techniques.

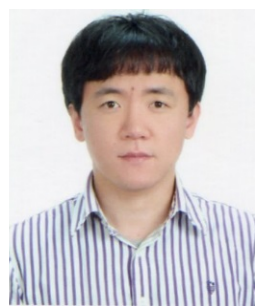

Deok Won Lim He is a senior research engineer of the Satellite Navigation Team at the Korea Aerospace Research Institute, Korea. He received B.S. and Ph.D. degrees from Chungnam National University. His current interests include multipath mitigation techniques and jammer localization systems.

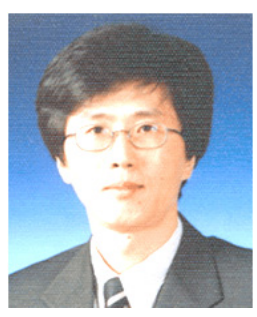

Sang Jeong Lee He is a Professor of school of Electronics Engineering of Chungnam National University, Korea. $\mathrm{He}$ received B.S., M.S. and Ph.D. degrees from Seoul National University. His current interests include digital signal processing and software GPS receiver.

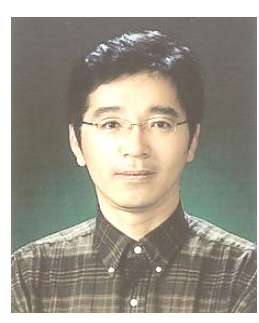

Chansik Park He is a Professor of school of Electronics Engineering of Chungbuk National University, Korea. He received B.S., M.S. and Ph.D. degrees from Seoul National University. His current interests include ambiguity resolution and the carrier phase navigation. 